\title{
Evolutionary Optimization of Business Process Designs
}

\author{
Ashutosh Tiwari, Kostas Vergidis and Rajkumar Roy \\ School of Applied Sciences, Cranfield University, \\ Cranfield, MK43 0AL, U.K.
}

\begin{abstract}
Summary. Business process redesign and improvement have become increasingly attractive in the wider area of business process intelligence. Although there are many attempts to establish a qualitative business process redesign framework, there is little work on quantitative business process analysis and optimization. Furthermore, most of the attempts to analyze and optimize a business process are manual without involving a formal automated methodology. Business process optimization can be classified as a scheduling problem, expressed as the selection of alternative activities in the appropriate sequence for the available resources to be transformed and thus satisfy the business process objectives. This chapter provides an overview of the current research about business process analysis and optimization and introduces an evolutionary approach. It demonstrates how a business process design problem can be modeled as a multi-objective optimization problem and solved using existing techniques. An illustrative case study is presented to demonstrate the results obtained through three multi-objective optimization algorithms. It is shown that multi-objective optimization of business processes is a highly constrained problem with fragmented search space. However, the results demonstrate a successful attempt and highlight the directions for future research in the area.
\end{abstract}

\section{Introduction}

Business process redesign is inherently linked to the scheduling problems. One of the main topics of research in scheduling has been the optimal allocation of resources to tasks. Business processes can be analyzed using

A. Tiwari et al.: Evolutionary Optimization of Business Process Designs, Studies in Computational Intelligence (SCI) 49, 513-541 (2007) 
similar perspective. The design and management of business processes is a key factor for companies to effectively compete in today's volatile business environment. By focusing on the optimization and continuous improvement of business processes, organisations can establish a solid competitive advantage by reducing cost, improving quality and efficiency, and enabling adaptation to changing requirements. Multi-objective optimization of business processes can result in novel approaches and more efficient ways of business process improvement as more than one optimization criteria can be selected and satisfied concurrently.

The rest of the chapter is organized as follows: Section 2 introduces the basic concepts of business processes and examines the basic formal definitions. Section 3 presents existing performance analysis techniques for business processes and section 4 investigates current optimization approaches for formal business process models. Section 5 presents the authors' approach towards evolutionary multi-objective optimization of business processes and section 6 introduces the results of this approach. Finally, section 7 discusses the potential directions for future research in the area.

\section{Formal Approaches to Business Processes}

For a business process design to be optimized, the model construction methodology plays a decisive role. This section provides an introduction to the 'business process' concept and presents the most common modeling notions to provide a familiarization with the basic ideas. The focus is to the business process modeling techniques that allow formal optimization. This section begins with a discussion on how different authors perceive business processes and how these are related to scheduling.

\subsection{Business Processes and Scheduling}

An overview of most common business process definitions is provided in [16] suggesting that most of the attempts to define a business process are inadequate and confined to a mechanistic viewpoint of the process. Most of these definitions ignore the human side resulting in static simplified representations of business processes [18]. According to [5], business processes are complicated and thus more difficult to be fully specified. However, the 'mechanistic' definitions of business processes bring them closer to scheduling problems thus making a range of successful approaches already applied to scheduling, available. 
There are many authors influenced by this perspective and provide business process descriptions that are more indicative of scheduling problem descriptions. For example, Hammer and Champy [9] suggest that a process is a 'set of partially ordered activities intended to reach a goal'. A process is thus a specific ordering of work activities across time and place with a beginning, an end and clearly identified inputs and outputs: a structure for action. A similar perception [3] also suggests that a business process can be perceived as a network of tasks. This approach is in line with scheduling, as it is concerned with resource allocation to tasks and justifies the attempt in [28] to map scheduling problems using a technique (i.e. Petri-nets) that has been already used for business process modeling.

Another attempt to define business processes and relate them to scheduling is found in [2] describing a business process as 'the combination of a set of activities within an enterprise with a structure describing their logical order and dependence, whose objective is to produce a desired result'. Also, in [11] the business process model described is quite similar to the Resource-Constraint Project Scheduling Problem (RCPSP). An inadequate attempt to define and model a business process could have a significantly negative effect on people's productivity by restraining their ability to gain expertise and apply innovative approaches while carrying out a business process.

\subsection{Business Process Modeling}

Business process modeling gives a snapshot of what is perceived at a point in time, in terms of a process that takes place in a business environment. Process models are currently best used to represent the internal elements of business processes; for example the activities needed and their dependencies, the dataflow, the roles and actors involved, and the goals. Along with the large number of attempts to define business processes, there is an abundance of techniques for capturing and modeling business processes. Aguilar-Saven [2] provides an overview of the most commonly used business process modeling techniques.

According to [17], business process improvement is dependent on an insight in the structure of business processes and their relations. This insight can be obtained by creating business process models that clearly and precisely represent the essence of the business organization. Business process modeling is itself a complex interdisciplinary and time-consuming process that involves judgments based on domain knowledge and experience, due to the multifaceted and dynamic nature of organizations [23]. The main objective of process modeling is the high-level specification of processes 
thus a process model should be properly defined, analyzed, verified, and refined to all of its aspects including structure, data flow, roles and constraints [24]. It is also important to identify the uses or purposes of the model when undertaking modeling of any kind in order to select the most appropriate technique [2]. Phalp [22] also highlights notation and method as two important parameters that need to be taken into account when modeling a business process as the model's general purpose and specific characteristics are significantly influenced by these two.

Taking the above into consideration, we can apply a number of criteria to classify the various modeling approaches. Frameworks and classifications of business process models can be found in relevant literature based on the purpose of the model, the notation or the structure ([2], [14]). We introduce a classification of business process models according to their formality, i.e. their ability to represent a process in a mathematically correct and rigorous way. The result of this classification is two groups of process models. On one hand are the so-called traditional or diagrammatic methods of process modeling, e.g. flowcharts or IDEF models and on the other hand, the business process modeling approaches that can be formally (i.e. mathematically or algorithmically) analyzed and verified. The next section introduces three representative formal business process modeling approaches. We choose to focus on these models because performance analysis and optimization - that is our main areas of focus- can be applied almost exclusively to this second group.

\subsection{Formal Modeling Approaches}

The most frequently recognized shortcoming of process modeling is the lack of analysis tools. Owing to the qualitative and static nature of most process models, mathematical techniques are difficult to apply. In order to make the process modeling methodologies more attractive, formal techniques for analysis of process models are required [30]. Formal process models are the ones in which process concepts are defined rigorously and precisely, so that mathematics can be used to analyze, extract knowledge from and reason about them. An advantage of formal models is that they can be verified mathematically, can be proved that they are consistent, and have or lack certain properties [15]. Although there are a number of formal modeling approaches, the majority of the business reengineering community uses simple diagrammatic modeling techniques [19]. This affects the analysis of business processes restricting it to simple inspection of the business process diagrams thus the conclusions are mostly heavily dependent upon the skill of the modeler. 
Luttighuis et al. [17] recommends formal methods for detailed analysis of process over the diagrammatic modeling. The same perspective is also highlighted in [2] when suggesting that the analysis of business processes needs models that present both the dynamic and functional aspects of the process and also sophisticated mechanisms that qualitative analysis of static diagrammatic models cannot offer. Although formal methods can provide significant benefits to business process modeling by introducing new perspectives, there is a lack of formal methods to support the actual design of business processes [11]. This is mainly because design elements and constraints on process designs are hard to characterize in a formal way amenable to analytical methods. The qualitative nature of process designs explains the difficulty of 'parametric' models of business processes [26]. Three representative formal business process models found in the literature are discussed below.

\subsubsection{Petri-nets}

Petri-nets is a formal graphical process modeling language. According to [10], Petri-nets help describe the semantics of process control flow, including basic branch and join rules, as well as more complicated synchronization scenarios. Petri-nets are an established tool for modeling and analyzing processes that has been widely recognized. They can be used as a design language for the specification of complex workflows and also Petrinet theory provides powerful analysis techniques that can be used to verify the correctness of workflow procedures - they can be used for both qualitative and quantitative analysis of workflows and workflow systems [29]. A Petri-net is a directed graph that uses as main constructs places, transitions, tokens and arcs.

- Places: drawn as a circle, a place is a stopping point in a process, the attainment of a milestone.

- Transitions: a transition is a rectangle that represents an event or action.

- Tokens: A token is a black dot residing in a place representing the current state of the process. During the execution of the process, tokens move from place to place.

- Arcs: An arc is a link from a transition to a place or a place to a transition.

Van der Aalst [29] supports that Petri-nets have a series of advantages that played a key role in their establishment. Although they support diagrammatic process modeling, they also provide formal semantics. Moreover, 
apart from process events, they record the various states of the process providing a more holistic view on the process flow. Also, they offer an abundance of analysis techniques that can be used to evaluate the performance of the modeled business process by calculating e.g. the estimated throughput of a process, the average throughput time of a job, the estimated occupation rate, etc. [27]

However some attempts to use Petri nets in practice reveal two serious drawbacks. First of all, there is no data concept and hence the models often become excessively large, because all data manipulation has to be represented directly in the net structure. Secondly, there are no hierarchy concepts, and thus it is not possible to build a large model via a set of separate sub-models with well-defined interfaces [2]. For these reasons Petri-nets have been extended supporting constructs like time and color.

\subsubsection{Business Process Al-based Language}

The second approach to formal business process modeling comes from [15]. The proposed business process modeling methodology is constructed with an Artificial Intelligence (AI) programming language thus ensuring the formality of the proposed process model.

The methodology begins with the definition of business process objectives. The output is a detailed formal specification of a business process that achieves those objectives. This perspective is established and confirmed by the logical assumption that a process model cannot be represented by a single model but as a set of various sub-models that capture the business process from different viewpoints. There are five interconnected sub-models specified to formally describe different aspects of the business process are:

- organizational sub-model, describing the actors that participate in the process, their roles, their responsibilities and their capabilities,

- objectives and goals sub-model, describing what the process and its actors try to achieve,

- process sub-model, describing how the process will achieve those goals,

- concepts sub-model, describing non-intentional entities, and

- constraints sub-model, describing factors limiting what the enterprise and its components can do.

Each of these models consists of various concepts that are formally described by the declarative logical language $L$ [7]. $L$ is used to introduce appropriate constructs, write axioms and capture process semantics. The em- 
phasis is on actor and role concepts as each role involves a set of responsibilities and actions that need to be carried out by an actor. $L$ is used here to precisely define the relationships between various concepts.

Using the same methodology, other sub-model concepts like goals and actions are specified. This approach ensures the formality of the concepts although it becomes increasingly complicated as the concepts to be modeled incorporate other concepts. This attempt can be discouraging because 'it is a lot of work to create and maintain a formal business process and also retain its consistency' [15]. Other disadvantages of this proposal lie in the use of complex mathematical notation that might put off the business analyst and the skills of AI programming that are essential but rarely found in an average manager.

\subsubsection{Scheduling-based Mathematical Formulation of Business Processes}

The third approach to formal business process modeling comes from [11] and it is related to mathematical definition of business processes. This modeling approach is linked with three different optimization approaches that will be thoroughly discussed later on this chapter. A business process is described using a mathematical model with an objective function which can portray any business process objective e.g. cost. The objective function is minimized or maximized by the optimization algorithm.

The main concepts used in the process design are activities and resources. A business process is perceived as a sequence of activities. These activities use some resources and produce others to be used by the following activities until the goal resources are produced. Resources are the physical or information objects which flow through the system. Activities are transformation steps which use resources as inputs and produce new ones as outputs. Both activities and resources are represented as sets. Each process begins with some input resources and produces a desired set of output resources. Each activity has two parameters: one for its starting time and another for its execution duration. The input resources of this activity must be available before the activity starts and the output resources must be produced after the activity has been executed. The time that a resource becomes available is another parameter critical to process feasibility.

The particular modeling approach gives flexibility to the authors, to apply different optimization methods. It is also close to scheduling problem definition as it explicitly involves activities and resources to a business process design. The process model is adjusted and further defined in each of the different optimization attempts. This approach will be more exten- 
sively discussed later as it forms the basis for our evolutionary optimization of business process designs.

\section{Business Processes Performance Analysis}

Qualitative performance analysis of business processes can only occur to formal business process models. It is the only kind of process analysis that can contribute to process optimization by identifying the process bottlenecks that can be optimized. A business process might be correct (verified) and also produce the expected outcome in a given context (validated) but it could still have redundant steps or not satisfactory performance in terms of cost effectiveness, duration, resource allocation etc. Before introducing the existing business process optimization approaches in the next section, this section provides a brief introduction to different process analysis attempts in literature that approach the subject from different perspectives and can be considered as a significant first step towards business process improvement.

\subsection{The Fuzzy Logic Approach}

Zakarian [30] attempts to model and quantify a business process using a combination of fuzzy logic and rule-based reasoning. The main motivation for this approach is to model efficiently the uncertain and incomplete information of process variables that exist in most of the traditional modelling techniques. The starting point of this approach is a business process illustrated with an IDEF3 model. This model is used as the basis for quantification and performance analysis of the business process. IDEF3 is quite popular and widely used modelling method in the business process context. One of the major advantages of IDEF3 representation is its simplicity and its descriptive power. The essence of IDEF3 methodology is its ability to describe activities and their relationship at various levels of detail, because an initial model includes parent activities that can be decomposed into lower level activities. These models are also easy to extend. IDEF3 offers several important characteristics for successful process representation:

1. process description in the form of activities,

2. structure of the underlying process, and

3. flow of objects and their relationship. 
In the first step of the process presented in [30], IF-THEN fuzzy rules are extracted from the IDEF3 model and thus the linguistic variables are defined. The linguistic variables, which owing to their nature contain incomplete or uncertain information, fall into two categories: input and output variables. In the formal model, which consists of IF-THEN fuzzy rules, the input variables appear in the IF part, while the output variables are found in the consequent THEN part. In order to understand the author's approach an example with two fuzzy rules is demonstrated with the assumption that the rules were extracted from a simple IDEF3 model:

- Rule 1: IF product stock is low THEN Marketing department informs customers about urgent orders.

- Rule 2: IF shipping products' weight is heavy THEN postage costs are high.

The linguistic variables for rule 1 are 'low' and 'urgent' and for rule 2 'heavy' and 'high' and they need to be quantified. The quantification will take place by assigning possibility distributions to the linguistic variables. Possibility distribution is the definition of the lower and upper limits of a fuzzy set using numbers. For example, 'low' product cost can be defined by the fuzzy set as being lower than 1,000 product items. The possibility distribution is precisely defined as the result of a process called 'defuzzification'. De-fuzzification is about obtaining a crisp value for each of the output fuzzy set variables (e.g. 'high post costs' can be de-fuzzified to 100 dollars).

The main outcome of this methodology is that having started from an IDEF3 model containing incomplete information about its linguistic variables, a set of specific IF-THEN rules is extracted based on the model's flow. The linguistic variables of these rules are then categorised into fuzzy sets which are de-fuzzified by assigning precise boundaries. The significance of this procedure is that the process is accurately executed and its output can be quantified and predicted. Performance analysis can then occur by combining different values for each variable to estimate the various process outputs.

This methodology was applied to a real industry process problem (film decomposition process) and succeeded in calculating process outputs for different values of the linguistic variables of the process. This provides the opportunity to perform performance analysis of the process output for different scenarios. This modelling and analysis approach, being co-operative (or supplementary) to an IDEF3 process model, manages to represent and quantify information that is usually not applicable to diagrammatic process models. The effectiveness of quantification is apparent as it can be used to 
combine a number of values for each participating variable and to produce potential process outputs helping the business analyst to track which process scenario is more beneficial.

\subsection{Quantifying Role Activity Diagrams}

Role activity diagrams (RADs) are based on a graphical view of the process from the perspective of individual roles, concentrating on the responsibility of roles and the interactions between them [12]. Roles are abstract notations of behaviour describing a desired behaviour within the organisation. RADs are object-state transition diagrams used in object-oriented models. They describe how a role object changes state as a result of the occurring actions and interactions.

Phalp [21] attempts to quantify RADs thus create a measure for performance analysis. The main objective of this approach is to minimise the coupling between the various roles in a Role Activity Diagram. Coupling is defined as the interaction between two or more roles in order to complete a task [20]. Coupling can be captured and visualised within RADs. The starting point for this coupling measure is the sum of actions and interactions of a particular role. This measure is called 'coupling ratio' $\left(\mathrm{C}_{\mathrm{p}} \mathrm{F}_{\mathrm{X}}\right)$ measuring the correlation between actions (independent activities of a role) and interactions (involvement of another role) in terms of percentage. If the ratio is high, it means that the coupling is large and the business process needs further improvement.

A major benefit of the coupling ratio is that it enables comparison between roles of different sizes [21]. Reducing coupling allows roles to become more autonomous because they do not need to synchronise. This has a major effect on the business process as it improves the performance in a way that each role performs its tasks more quickly and with less opportunity to delay since there is independence from redundant interactions. This approach enables business process performance analysis by quantifying one feature, i.e. role coupling. Reducing coupling in a process, directly results in the process becoming more straight-forward, faster and-in general terms- improved through the analysis and inspection of the coupling ratio.

\subsection{Performance Analysis through a Query Language}

The last business process performance analysis approach is workflow related. Workflow is a similar concept to business process and many authors are using these two keywords interchangeably. In [1] an approach is proposed for the performance evaluation of automated business processes 
based on the measurement language WPQL (Workflow Performance Query Language). The adoption of a Workflow Management System (WfMS) to automate a business process gives the opportunity to collect real execution data continuously, from which exact information about the process performance can be obtained. On the one hand, such data can be used for monitoring, work balancing and decision support. On the other, execution data can feed simulation tools that exploit mathematical models. Through the simulation, it is possible to obtain an assessment of the current process performance and to formulate hypotheses about possible reengineering alternatives.

Although formal languages have been exploited in order to define the concept of process, the use of formal languages to handle the problem of performance evaluation of workflow has received not as much coverage [1]. A language is an application tool that could enable the writing of queries against a WfMS in order to compute measures about given workflow entities. The main benefit of such a tool is the expressive power of a programming language to define and evaluate new performance measures. The basic idea of the WPQL language is based on the following steps:

1. Define a new measure

2. Select workflow entities to measure

3. Apply the measure to the selected entities

The WPQL also offers a standard set of performance measures as well as the facilities to define new ones. WPQL is a specialized language, its focus is on the measurement of workflow related quantities and its constructs have been introduced to ease the handling of concepts such as process or workflow participant.

\section{Scheduling-based Business Process Optimization Approaches}

After reviewing the business process modeling techniques and performance analysis approaches, it is time to examine the not-as-many business process optimization attempts. Process improvement - often used as an umbrella term for performance analysis and business process optimizationis one of the most significant motivations for process modeling. According to [25], large organizations are attempting to map their processes for two main reasons: One is to acquire a realistic knowledge of the current situation and flow of activities within the organization and second to efficiently improve those processes thus meeting the organization goals. The prereq- 
uisites for business process optimization are: (i) the business processes should be correctly designed, (ii) their execution should be supported by a system that can meet the workload requirements, and (iii) the (human or automated) process resources should be able to perform their work items in a timely fashion [8]. The same would stand for any other scheduling-type of problem.

The quality of a business process is defined by many, often conflicting criteria such as costs, duration, or quality of output. A business process optimization approach should clearly define and specify how optimization is perceived and which aspects of the process are going to be optimized. Not many optimization techniques found in literature are suitable for business processes. Due to their qualitative nature, process designs are hard to characterize in a formal way amenable to analytical methods and thus improve them in a measurable way. There is also a lack of tools for identifying the bottleneck areas in these models. Their qualitative nature also explains the difficulty of developing 'parametric' models of business processes. For these reasons, there is a lack of algorithmic approaches for the optimization of business processes [26]. In this section some optimization techniques found in literature are discussed.

\subsection{Mathematical Programming Formulation}

As mentioned previously, there is an attempt in [11] to optimise a business process using three different approaches that are examined thoroughly in this section. Having already mentioned the modelling approach towards business processes it becomes easier to understand the perspective towards the model's optimization.

The formulation of the process design as a mathematical problem is close to scheduling problems. A linear objective function and a number of constraints are used to describe the problem and cover all of its aspects. The objective function -which can illustrate a process objective e.g. costis minimised or maximised according to the goal of optimization. The constraints describe and ensure the feasibility of the process in a mathematically formal way. A constraint, for example, can restrict the use of a particular resource before it is produced within the process. The main concepts used in the process design are activities and resources. The mathematical constraints regarding the activities and resources can be grouped into two major categories:

1. constraints related to input and output resources of each activity and

2. constraints regarding the sequence and timing of resources and activities. 
Each process begins with some input resources $\left(\mathrm{I}_{\mathrm{glob}}\right)$ and produces a desired set of output resources $\left(\mathrm{O}_{\text {glob }}\right)$. The participating activities should be sequenced in such a way that they use some resources as inputs and then produce resources that can be used as inputs by other activities until the desired output is produced. Constraints that belong to the first group make sure that input resources are available by activities to use and that the final set of output resources is eventually produced. The second group of constraints checks the time sequence of activities and resources. Each activity has a starting time $p$ and an execution duration $d$. The input resources of this activity must be available before $p$ and the output resources must be produced in $p+d$ time. The time that a resource becomes available is $q$ and is critical to the feasibility of the process.

In order to formally set the constraints, a number of variables and arrays that bind together the resources and activities are being introduced. This increases the complexity of the process model but also ensures its mathematical formality. It also makes the model more flexible as a constraint can be eliminated to simplify a particular aspect of the model or extra constraints can be inserted to further shape the model. The total number of constraints in the final mathematical is thirteen. According to experiments [11] the mathematical approach produced satisfying results but high execution times.

\subsection{Direct Branch and Bound Method}

Apart from mathematical programming, branch and bound method is another way of optimising a business process [11]. Optimization problems that have a significant number of binary variables are often solved using branch and bound algorithms as they are easy to implement. Moreover, these algorithms provide a bound on the optimal objective during execution, meaning that they can be stopped when the potential improvement is small, without solving the problem to full optimality. During the branch and bound procedure, a search tree is generated and in every branching step another activity is added to the tree constructing the process design i.e. at the first level, the first activity to be included in the design is selected, at the second level the second activity and so on. When a feasible solution is found then the particular node is no longer expanded if it contains a feasible solution. In order to implement a branch and bound strategy, two particular problems are being examined [11]:

1. The selection of a node to be expanded.

2. The computation of bounds on the objective value at each node. 
In terms of the first problem, two strategies attempt to restrict the set of activities considered for branching: (i) The forward strategy that starts from global inputs using resources available to link activities and produce the global output and (ii) the backward strategy that starts from global outputs and tries to generate initial resources. Computational experiments confirm that the forward strategy is considerably more efficient. These experiments show that the branch and bound algorithm turned out to be considerably fast, solving most of the problems quickly and yielding better results than other approaches.

\subsection{Genetic Algorithms}

Genetic Algorithms (GAs) has been a popular method that has been developed and successfully applied to complex problems in a variety of areas. A significant advantage of GAs is that they maintain a population of possible solutions to reach feasibility and that makes them more powerful. Another significant advantage is their extendibility to optimise a problem with more than one objective. Multi-objectivity makes genetic algorithms a flexible and truly beneficial methodology that can be applied to any optimization problem.

In order to find an optimal solution, a genetic algorithm imitates the process of natural evolution. It works on a large number of solutions in parallel, where each solution corresponds to an individual in a population. Each solution is represented by an appropriately coded string. Initially, a set of randomly generated solutions is produced. Then, superior solutions are selected to form a new population. The selection probability depends on the objective function value. The resulting selected individuals are then selected for mating. A crossover operation exchanges information between two individuals. Finally, a mutation operation changes the values of randomly chosen bits. This process continues until some pre-defined termination criteria are fulfilled.

The business process design that is proposed in [11] has a significant number of constraints. There are two different approaches to deal with constraints in the GAs optimization approach. In the first approach, a penalty term for constraint violation is added to the original objective function. The second approach modifies the genetic operators to limit the search space to feasible solutions. This approach is appropriate if feasible changes can easily be determined. Nevertheless, during the performance tests, genetic algorithms show weak performance. Their main problem is the feasibility maintenance in this business process design problem. Our approach -presented on the next section- attempts to resolve these prob- 
lems by modifying the business process model appropriately and then applying selected evolutionary algorithms to achieve multi-objective optimization.

\section{Scheduling-based Evolutionary Multi-objective Optimization}

This section introduces our approach towards business process optimization. A formally defined business process model is optimized using evolutionary algorithms. The utilization of evolutionary techniques provides the additional ability of optimizing the problem under multiple criteria. The framework to be presented next, aims to introduce a methodology for applying multi-objective optimization algorithms to business process models. It consists of two main stages: The first stage of the framework is the business process model definition and the second stage involves the application of the evolutionary algorithms to a test business process model for optimization results generation.

\subsection{Business Process Model}

The first stage of the optimization framework is the business process model specification. The model has a mathematical basis to ensure formality, consistency and rigor. The business process model is limited to a series of mathematical constraints that define the feasibility boundaries of the business process and a set of objective functions that consist of the various business process objectives. Representing a business process using a formal mathematical model guarantees the construction of consistent and rigorous business processes following a formally correct, repeatable and most importantly, verifiable approach [15].

The business process model consists of two key concepts: activities and resources. Apart from the resources that are generated within the process, the business process design has two sets of resources, the initial $\left(\mathrm{I}_{\mathrm{glob}}\right)$ and the final $\left(\mathrm{O}_{\mathrm{glob}}\right)$ resources. The initial resources are available at the beginning of the business process, while the final resources form the business process final output. The resources flow through the process and belong to two categories: physical and information resources. The activities, on the other hand, are perceived as the transformation steps within the process that use some resources as inputs and produce others as outputs. In a feasible process design, all the activities are in a defined sequence, the avail- 
ability of resources is adequate and most importantly the final (output) resources are being produced by the participating activities.

For the business process model to be optimised, it is necessary to define the process objectives (e.g. process duration) as well as the input variables (e.g. activity duration). The business process model receives as input variables the participating activities and their starting times, whereas the aim is to produce an optimised process in terms of minimising two objectives, the process duration and cost. For each process design, there is a library of candidate activities with attributes such as activity duration and activity cost. For an optimised business process design to be produced, a set of activities that generate minimum business process cost and duration needs to be selected. It is important to note that the framework works independently of the number of objectives and their type. Process duration and are cost chosen as the two objectives for business process improvement. The complete mathematical model is the following:

$f_{1}(P)=\max \left(q_{j}\right) \rightarrow \min , \forall j: b_{j} \in g o_{j}$

$f_{2}(P)=\sum u_{i 1} x_{i} \rightarrow \min$

s.t.

1. $x_{i} \leq r_{i j}, \forall i, j: b_{j} \in I_{i}, b_{j} \in B_{P}$,

2. $x_{i} \leq y_{j}, \forall i, j: b_{j} \in I_{i}, b_{j} \in B_{I}$,

3. $g o_{i}+\sum_{i} r_{i j} \leq M \cdot g i_{j}+\sum_{i} t_{i j} x_{i}, \forall j: b_{j} \in B_{P}$,

4. $y_{j} \leq g i_{j}+\sum_{i} t_{i j} x_{i}, \forall j: b_{j} \in B_{I}$,

5. $y_{j} \geq g o_{j}$,

6. $p_{i} \geq q_{j}-M\left(1-x_{i}\right), \forall i, j: b_{j} \in I_{i}$,

7. $q_{j} \leq p_{i}+\delta_{i}+M\left(1-x_{i}\right), \forall i: b_{j} \in O_{i}$

8. $q_{j} \geq p_{i}+\delta_{i}-M\left(1-x_{i}\right)-M\left(1-\lambda_{i j}\right), \forall i: b_{j} \in O_{i}$,

9. $\lambda_{i j} \leq x_{i}, \forall i, j: b_{j} \in O_{i}$,

10. $\sum_{i: b_{j} \in O_{i}} \lambda_{i j} \geq \sum_{i} r_{i j}+o g_{i}-M\left(1-y_{j}\right), \forall j: B_{P}, g i_{j}=0$,

11. $\sum_{i: b_{j} \in O_{i}} \lambda_{i j} \geq 1-M\left(1-y_{j}\right), \forall j: b_{j} \in B_{P}, g i_{j}=0$,

12. $x_{i} \in\{0,1\}, \forall i$,

13. $\lambda_{i j} \in\{0,1\}, \forall i, j: b_{j} \in O_{i}$. 
The mathematical model of business process defines the optimization objectives with two objective functions and ensures the business process consistency and feasibility with thirteen constraints. Further objectives can be added with extra functions. The process model appears to be complicated in contrast to the simplistic approach of the business process consisting only of activities and resources. A brief description of the mathematical model's main features can provide a good understanding of its functionality. The mathematical model consists of a number of binary variables and binary matrices that have an impact on the production of feasible process designs since they result to a highly fragmented search space. The first objective function $\left(f_{1}\right)$ of the model calculates the duration of the business process. The total duration for a feasible process equals the time the last resource that belongs to global outputs is produced. The second objective function $\left(f_{2}\right)$ calculates the business process cost as the sum of costs of all participating activities.

Table 1. Main Parameters in Mathematical Model

\begin{tabular}{|c|c|}
\hline Parameter & Explanation \\
\hline$\overline{u_{i 1}}$ & Cost of execution for activity $a_{i}$. \\
\hline $\mathrm{X}_{\mathrm{i}}$ & $\begin{array}{l}\text { Binary variable that indicates whether a candidate activity } a_{i} \text { par- } \\
\text { ticipates in the business process design. }\end{array}$ \\
\hline $\mathrm{y}_{\mathrm{j}}$ & $\begin{array}{l}\text { Binary variable that indicates whether resource } b_{j} \text { is or becomes } \\
\text { available in the business process. }\end{array}$ \\
\hline$t_{i, j}$ & $\begin{array}{l}\text { Matrix of binary variables that link the activities with their out- } \\
\text { put resources. }\end{array}$ \\
\hline$r_{i j}$ & $\begin{array}{l}\text { Matrix of binary variables that indicate if a unit of physical re- } \\
\text { source } b_{j} \text { is available for use by activity } a_{i} \text {. }\end{array}$ \\
\hline $\mathrm{gi}_{\mathrm{j}} \& \mathrm{go}_{\mathrm{j}}$ & $\begin{array}{l}\text { One-dimensional binary constants that indicate which resources } \\
\text { belong to global inputs and/or global outputs. }\end{array}$ \\
\hline M & $\begin{array}{l}\text { Large constant indicating that physical resources contained in } \\
\text { the set of global inputs are available in unlimited amounts. }\end{array}$ \\
\hline$p_{i}$ & Starting time of activity $\mathrm{a}_{\mathrm{i}}$ \\
\hline $\mathrm{q}_{j}$ & Time when the resource $b_{j}$ becomes available. \\
\hline$\delta_{\mathrm{i}}$ & Duration of activity $a_{i}$. \\
\hline$\lambda_{\mathrm{ij}}$ & $\begin{array}{l}\text { Binary variable indicating that activity } a_{i} \text { is used to create re- } \\
\text { source } b_{j} \text {. }\end{array}$ \\
\hline $\begin{array}{l}\mathrm{I}_{\mathrm{i}} / \mathrm{O}_{\mathrm{i}} \\
\mathrm{B}_{\mathrm{p}} / \mathrm{B}_{\mathrm{H}}\end{array}$ & $\begin{array}{l}\text { Sets of input/output resources of activity } a_{i} \text {. } \\
\text { Set of physical / information resources } b_{j} \text {. }\end{array}$ \\
\hline & \\
\hline
\end{tabular}

The mathematical model constraints ensure that the model produces feasible business processes by examining different aspects of the business process model. Table 1 provides an explanatory legend for all the mathematical model variables and parameters and table 2 provides a short 
description of each constraint of the mathematical model. It is also important to highlight two features of the business process model. The mathematical model consists of many discrete binary variables that significantly increase the complexity of even a simple process design as the search space for feasible solutions is highly fragmented. Another feature of the business process model is that although it is simple to conceive and understand, it is highly constrained when it comes to formal mathematical definition. This can create serious difficulties in locating the optimum solutions since even feasible solutions are hard to be produced. The concepts that describe the business process and its mathematical model are inspired by [11]. Our aim is to extend the model to multi-objectivity and optimise it using multiobjective evolutionary algorithms.

\subsection{Test Problem Construction}

This section describes the construction of a business process design test problem. Five different test process designs were constructed for assessing their capability to be optimised by the optimization algorithms. We will describe analytically the construction of one of these problems.

The test problems utilised, have an increasing number of activities participating in each process design. The results demonstrate that this is a significant cause in increasing the problem complexity and has a serious impact on the quality of results. Each of the problems has a fixed predefined number of participating activities in the process. The initial and final resources of the business process are given. Remember that for each process design there is a library of candidate activities that can potentially participate in the process.

A business process design is optimised when the activities selected along with their starting times produce a business process with minimum duration and cost in contrast with any other combination of candidate activities. The case study discussed here is based on the generic business process model, it is called ActivitiesST4 and it involves four participating activities. The library of candidate activities contains 10 activities that can be alternatively used in various combinations of four. For ActivitiesST4 design to be optimised, the four activities selected to participate must minimise the total process cost and duration. 
Table 2. Explanation of Constraints

1. $x_{i} \leq r_{i j}, \forall i, j: b_{j} \in I_{i}, b_{j} \in B_{P}$

All input physical resources of an activity must be available $\left(\mathrm{r}_{\mathrm{ij}}=1\right)$ at some stage of the process if the activity is participating $\left(\mathrm{x}_{\mathrm{i}}=1\right)$.

2. $x_{i} \leq y_{j}, \forall i, j: b_{j} \in I_{i}, b_{j} \in B_{I}$

All input information resources $\left(\mathrm{y}_{\mathrm{j}}\right)$ of an activity must be available at some stage of the process if the activity is participating $\left(\mathrm{x}_{\mathrm{i}}=1\right)$.

3. $g o_{i}+\sum_{i} r_{i j} \leq M \cdot g i_{j}+\sum_{i} t_{i j} x_{i}, \forall j: b_{j} \in B_{P}$

The output physical resources -final or not- must not exceed the sum of initial and produced -during the process.

4. $y_{j} \leq g i_{j}+\sum_{i} t_{i j} x_{i}, \forall j: b_{j} \in B_{I}$

An information resource $\left(y_{j}\right)$ can be available either at the beginning of the process -as initial resource $\left(\mathrm{gi}_{\mathrm{j}}\right)$ - or as an output resource of a participating activity.

5. $y_{j} \geq g o_{j}$

A resource $\left(y_{j}\right)$ cannot be part of the output without first being available at some stage of the process $\left(\mathrm{go}_{\mathrm{j}}\right)$.

6. $p_{i} \geq q_{j}-M\left(1-x_{i}\right), \forall i, j: b_{j} \in I_{i}$

In terms of time, a participating activity must start $\left(\mathrm{p}_{\mathrm{i}}\right)$ only after the time that all its input resources have become available.

7. $q_{j} \leq p_{i}+\delta_{i}+M\left(1-x_{i}\right), \forall i: b_{j} \in O_{i}$

8. $q_{j} \geq p_{i}+\delta_{i}-M\left(1-x_{i}\right)-M\left(1-\lambda_{i j}\right), \forall i: b_{j} \in O_{i}$

In terms of time, an output resource must become available exactly when the generating activity has been completed $\left(\mathrm{q}_{\mathrm{j}}=\mathrm{p}_{\mathrm{i}}\right)$.

9. $\lambda_{i j} \leq x_{i}, \forall i, j: b_{j} \in O_{i}$

A non-participating activity $\left(\mathrm{x}_{\mathrm{i}}=0\right)$ cannot have output resources $\left(\lambda_{\mathrm{ij}}=1\right)$.

10. $\sum_{i: b_{j} \in O_{i}} \lambda_{i j} \geq \sum_{i} r_{i j}+o g_{i}-M\left(1-y_{j}\right), \forall j: B_{P}, g i_{j}=0$,

When a physical resource does not belong to initial resources, it must be produced in greater or equal amounts to the required resource inputs.

11. $\sum_{i: b_{j} \in O_{i}} \lambda_{i j} \geq 1-M\left(1-y_{j}\right), \forall j: b_{j} \in B_{P}, g i_{j}=0$

Each physical resource that does not belong to initial resources but appears in the output of a participating activity must be produced at least once.

12. $x_{i} \in\{0,1\}, \forall i$

The variable $\mathrm{x}$ (indicating participating activities) must be binary.

13. $\lambda_{i j} \in\{0,1\}, \forall i, j: b_{j} \in O_{i}$

The variable $\lambda$ (indicating output resource $j$ of activity $i$ ) must be binary. 
The process design sketch of ActivitiesST4 problem is demonstrated in figure 1. Process optimization depends on the input parameters:

1. The appropriate activities need to be selected from the library and combined according to their duration and cost attributes and

2. The activities' starting times need to be properly calculated in order for the process outputs to be produced as early as possible and thus minimise the total process duration.

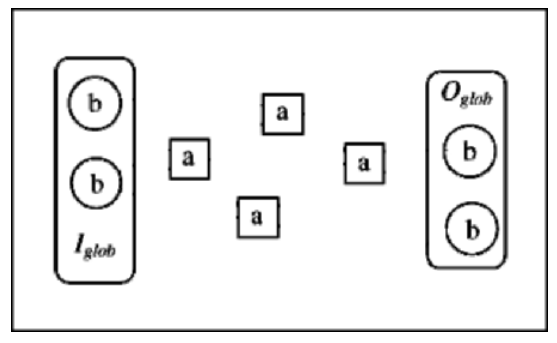

Fig. 1. ActivitiesST4 initial process design

The process design of figure 1 can be described as follows: There are two global input resources to start the process. These two resources together with the two global outputs are considered as constants. The system variables of the problem are the four participating activities and their starting time attribute. This means that the optimization algorithms attempt to meet the optimization objectives by defining a set of four activities (from a library of 10 alternatives) and the starting time for each of them. All the potential activities are stored in a built-in library and the algorithms can select any four activities. The four potential activities of the process design must be combined in a way that the given process output resources are produced. The optimization criteria are the minimisation of process duration and cost.

The other four test problems have identical structure as the one described. Their major differences are in the number of activities that participate in the business process design and the size of the library of candidate activities. The test process designs range from simple ones (e.g. ActivitiesST2 with two participating activities and a library of 10) to more complex ones (e.g. ActivitiesST5 with 5 activities in the process design and a library of 20 alternatives). As is mentioned earlier in this section, although the different test problems give the impression of having minor differences in the process size and library, these have a significant impact on the optimization performance as is demonstrated on the next section. 


\subsection{Experimental Results}

This section describes the experimental results for the test problem of the previous section. Three popular evolutionary algorithms that allow multiobjective optimization have been selected to optimise the business process model. The optimization algorithms that were selected are NSGA2, SPEA2 and MOPSO. These algorithms attempt to optimize the process designs by selecting different sets of activities and defining their starting times. Non-dominated Sorting Genetic Algorithm II (NSGA2) is nondominated, sorting-based, multi-objective evolutionary algorithm [6]. NSGA2 has been quite popular and has been applied to many problems on a number of research areas. Strength Pareto Evolutionary Algorithm II (SPEA2) is another elitist evolutionary algorithm with a fine-grained fitness assignment strategy, a density estimation technique, and an enhanced archive truncation method [31]. SPEA2 has also been quite popular and used in a variety of optimization problems. Multi-Objective Particle Swarm Optimization (MOPSO) is different from most evolutionary computation techniques as it is an extension of the Particle Swarm Optimization (PSO) method. MOPSO is demonstrating better performance in problems that have continuous search space [13]. Since more than one optimization methods are applied to the business process model, the opportunity of comparing the performance of the different algorithms in the particular problem context is appealing. NSGA2 and SPEA2 are being demonstrated in a number of papers and although similar they battle each other in quality of results in different subject domains. MOPSO on the other hand has never been used in such a constrained problem.

All the five test problems are optimised with each of the evolutionary algorithms. To evaluate the results a metric is also introduced which demonstrates the algorithms' performance. The 'success ratio' is the opposite of error ratio [6] and is calculated as the percentage of generated solutions that belong to the Pareto optimal front against the total number of solutions. The equation of the success ratio is:

$s_{R}=\frac{\text { no_of_solutions } \in P^{*}}{\text { total_solutions }} \%$

The numerator of the success ratio holds the number of generated solutions that belong to $\mathrm{P} *$ (Pareto optimal front) while the denominator holds the total number of generated solutions. 


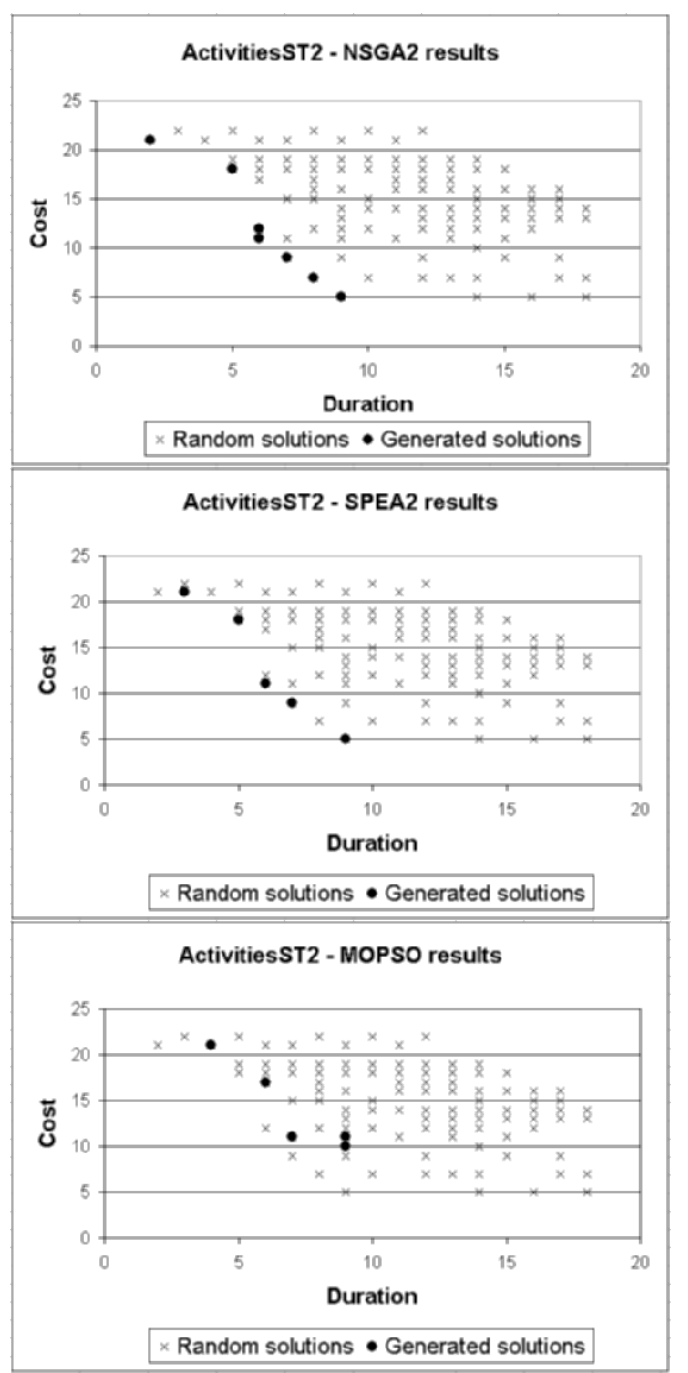

Fig. 2. Generated solutions for ActivitiesST4 by the optimization algorithms

In this particular context, the success ratio $\left(\mathrm{S}_{\mathrm{R}}\right)$ calculates the percentage of the near-Pareto optimal solutions that the optimization algorithm has generated because being a real-life situation the actual Pareto optimal front is not known for the test problem. To acquire a picture of the search space 10,000 random solutions were created and the feasible solutions amongst these were identified and plotted. Therefore, near-Pareto optimality of a 
solution in this case is defined with respect to the large set of randomly generated solutions such as the ones demonstrated in figure 2. A solution generated by an algorithm is considered here as near-Pareto optimal if it is non-dominated with respect to the set of these random solutions.

The test problems are incorporated in KEA toolbox [4] an optimization platform that uses (among others) NSGA2, SPEA2 and MOPSO algorithms to optimise user-defined problems. In order to produce the results, each of the optimization algorithms was executed 30 times with different random seed values. Most of these 30 runs produced similar results. The results presented here belong to one of those runs. The graphs in figure 2 demonstrate the solutions that each of the optimization techniques generated for the test process design. These solutions consist of feasible business processes with minimised process duration and cost. The graphs depict the process duration and cost values for both the random population and the optimised. The dotted points represent the solutions of each technique while the ' $x$ points' the random solutions. Each graph demonstrates the results for ActivitiesST4 process design by NSGA2, SPEA2 and MOPSO algorithms.

The success ratio was used to evaluate the results that the optimization algorithms produced. Figure 3 demonstrates the success ratio percentages for all the five test problems. For ActivitiesST2 process design, both NSGA2 and SPEA2 performed very well, unlike MOPSO that identified only $40 \%$ of the near-Pareto optimal solutions. SPEA2 also produced very good results for ActivitiesST3 problem, while NSGA2 gave a satisfactory number of optimum solutions. Nevertheless the algorithms' performance drops significantly with the addition of an extra activity in ActivitiesST4. MOPSO performs poorly as apart from the first test problem it does not seem to be able to locate optimum solutions. Moving to test problems with bigger activity libraries, NSGA2 produced satisfactory results for ActivitiesST4(20) only, while for ActivitiesST5 problem none of the algorithms was able to locate solutions near the Pareto front. The average success ratio for both NSGA2 and SPEA2 is approximately $40 \%$, while for MOPSO is only $8 \%$.

Before the results are further discussed, the features of the search space need to be highlighted once more as they seriously influence the quality of the results. The mathematical model of the business process designs consists of discrete binary variables that increase the optimization complexity even for a simple process design as the search space for feasible solutions is highly fragmented. Also the business process models are highly constrained having 13 constraints to check for every possible set of solutions, decreasing the performance of the algorithms. 
The optimization algorithms have a difficult task even to produce sets of feasible solutions. Given the complex nature of the business process design problem, the overall performance of NSGA2 can be characterised as good and can be attributed to its elitism. As NSGA2 archives the optimum solutions of each generation and compares them with the ones it produces, it manages to preserve the identified feasible solutions. SPEA2 is also an elitist algorithm that provides bigger spread of the solutions. It also preserves feasible individuals through generations and that justifies its satisfactory results. On the other hand, MOPSO seems to have a serious problem in successfully defining the guide that combines the two objectives. The algorithm demonstrates poor performance as the solutions that are generated are not near to the Pareto optimal front for most of the test problems. This supports the claim that MOPSO has best performance in problems with continuous search space [13] which is not the case here.

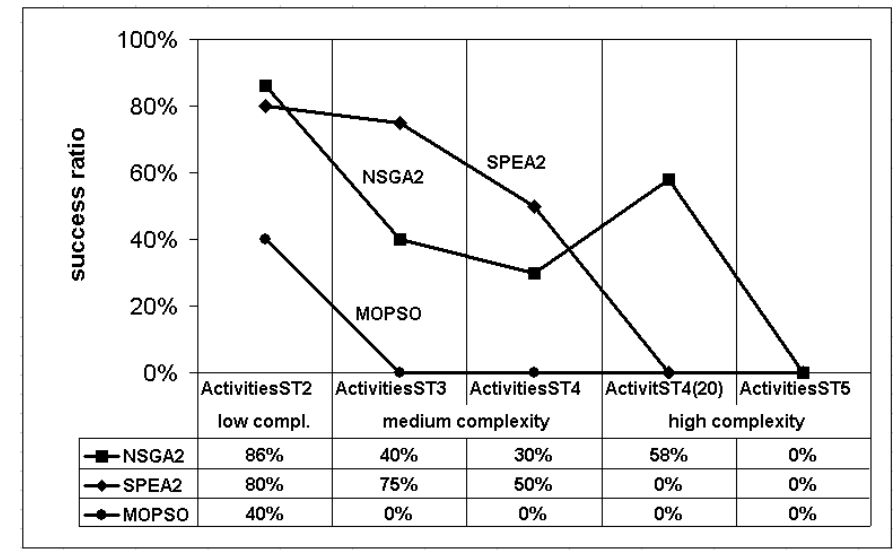

Fig. 3. Evaluation graph based on success ratio

Figure 3 also shows that as the complexity of the problems increases, the performance of the optimization algorithms declines significantly. The simplest of test problems (ActivitiesST2) is handled well by all three algorithms. Moving to medium complexity problems, SPEA2 provides better results while NSGA2 hits back on high complexity problems with $58 \%$ success on one of the problems. On average performance NSGA2 holds the best position with slightly better results that SPEA2 which has also performed above $40 \%$ on average. This enhances the view of SPEA2 and NSGA2 behaving very similar on various problems [31]. Many applications of the NSGA2, SPEA2 and MOPSO are not as successful in dealing 
with large dimensional problems and extremely disconnected Pareto fronts.

\section{Discussion of Results}

This section discusses the practical implications of the business process optimization framework, along with its limitations. As mentioned in a previous section, there are not many optimization techniques for business processes. Many of the techniques only provide quantifiable measures from diagrammatic process models.

However, the test problems demonstrated that the proposed framework is capable of successfully applying multi-objective optimization to various business process designs. The ability to produce an overall $40 \%$ of optimum solutions provides a good set of optimised alternative business processes with trade-offs in process duration and cost. This gives the capability to the business analyst to select a business process from a range of nearPareto optimal solutions according to decision making priorities. This extends the approach beyond a single objective. The results are indicative but also promising, and future research can lead to better quality results.

During the development of the multi-objective optimization methodology a number of limitations were unveiled. The first limitation originates from the mathematical model of the business process. The mathematical model focuses on activities and resources as its two main concepts and it ignores the participating (physical or mechanical) actors. This consequently results in what is criticised as 'a mechanistic viewpoint of business processes' [16]. Also it is more difficult for a formal business process modelling technique to capture the roles of the participants than a diagrammatic approach which visualises the flow of the process.

Another limitation lies in the selection of the five process designs as test problems. In order to better assess the optimization techniques used, an approach with a scalable range of problems was selected. Evaluating the algorithms' performance using a larger series of problems can better demonstrate the algorithms' behaviour providing a better and more apparent performance overview. The last limitation is linked with the evaluation metric that did not take into account the diversity of the generated solutions.

Formal business process optimization techniques can significantly contribute to the wider area of business process improvement in a number of ways. Firstly, an analytical method which takes into account the entire range of possible designs might produce process designs that are over- 
looked or cannot be conceived by a human designer. Secondly, by optimizing a process for different design criteria, the inherent trade-offs and the sensitivity of results to variations in design parameters will become more transparent with an analytical method. This can help a designer in identifying those parameters that are most important in achieving the desired goals. Therefore, business process optimization based on mathematical or algorithmical techniques can contribute significantly to introducing new perspectives and approaches.

\section{Directions for Future Research}

Future research in this area should focus on building more complete process models, testing more complicated process designs and exploring more efficient metrics. The construction of a business process model that can cover more aspects of a 'closer to real world' business process can be very useful for effective business process optimization. Business processes in real world have patterns such as feedback loops or decision points. Modelling and optimizing these aspects can prove a complicated process with increased complexity. Future research should also focus on selecting the most appropriate techniques for business process multi-objective optimization from a wider set of techniques and algorithms, and thus locating more accurately the most suitable optimization method. To improve the optimisation results of similar problems, there is a need to develop novel initialization and recombination schemes instead of relying to existing evolutionary approaches. Again, scheduling domain can provide techniques that have been successfully applied to solve complex scheduling problems of similar nature.

\section{Conclusions}

This chapter presented business process re-design as a problem of similar nature to scheduling. Business process optimization was perceived as the combination and sequencing of resources and alternative activities. After examining the business process concept in a generic sense and examining a selection of business process modeling, performance analysis and optimization techniques, we presented a framework for applying multi-objective optimization to business processes. By developing a formal business process model and orienting it to multi-objectivity, the generation of optimized business processes was facilitated. 
The business process optimization problem is unique because of its highly constrained nature and the fragmented search space that have a significant impact on locating the optimum solutions. It is shown that stateof-the-art multi-objective optimization algorithms, such as NSGA2 and SPEA2, produce satisfactory results by managing to generate and preserve optimal solutions. This provides a number of alternative optimised process designs for the business analyst to decide the trade-offs between the different objectives. The results presented here demonstrate that principles of scheduling could be effectively applied for optimization of business processes. This work is encouraging for further research in the area of business process multi-objective optimization.

\section{References}

[1] Abate AF, Esposito A, Grieco N, Nota G (2002) Workflow Performance Evaluation Through WPQL. In: Proceedings of the 14th International Conference on Software Engineering and Knowledge Engineering, Vol. 27. ACM Press, New York, pp 489-495

[2] Aguilar-Saven RS (2004) Business process modeling: Review and framework. Int J of Production Economics 90: 129-149

[3] Aiello R, Esposito A, Nota G (2002) A Hierarchical Measurement Framework for the Evaluation of Automated Business Processes. Int. J. of Software Eng. and Knowledge Eng. 12: 331-361

[4] Bartz-Beielstein T, Mehnen J, Naujoks B, Schmitt K, Zibold D (2004) KEA - A software package for development, analysis and application of multiple objective evolutionary algorithms, $<$ http://www.isf.maschinenbau. uni-dortmund.de/veroeff/documents/ 2004_mehn_kea_a.pdf> viewed February 2, 2006

[5] Curtis B, Kellner M, Over J (1992) Process modeling. Communications of the ACM 35: 75-90

[6] Deb, K.: Multi-objective optimization using evolutionary algorithms. John Wiley \& Sons, New York (2001)

[7] Enderton, H.B.: A Mathematical Introduction to Logic. Academic Press, New York (1972)

[8] Grigori D, Casati F, Castellanos M, Dayal U, Sayal M, Shan M.C. (2004) Business Process Intelligence. Computers in Industry 53: 321-343

[9] Hammer M, Champy J (1993): Re-engineering the Corporation: A manifesto for business revolution. Harper Business, New York

[10] Havey M (2005) Essential Business Process Modeling. O'Reilly, U.S.A

[11] Hofacker I, Vetschera R (2001) Algorithmical approaches to business process design. Computers \& Operations Research 28: 1253-1275

[12] Holt AW, Ramsey HR, Grimes JD (1983) Coordination systems technology as a programming environment. Electrical Communication 57: 307-314 
[13] Kennedy J, Eberhart R (1999) The Particle Swarm: social adaptation in information-processing systems. In: Corne D, Dorigo M, Glover F, (Eds.): New Ideas in Optimization. McGraw-Hill, Cambridge, pp 379-388

[14] Kettinger WJ, Teng JTC, Guha S (1997) Business Process Change: A Study of Methodologies, Techniques and Tools. MIS Quarterly 21: 55-80

[15] Koubarakis M, Plexousakis D (2001) A formal framework for business process modeling and design. Information Systems 27: 299-319

[16] Lindsay A, Downs D, Lunn K (2003) Business processes - attempts to find a definition. Information and Software Technology 45: 1015-1019

[17] Luttighuis PO, Lankhorst M, van de Wetering R, Bal R, van den Berg H (2001) Visualizing Business Processes. Computer Languages 27: 39-59

[18] Melao N, Pidd M (2000) A conceptual framework for understanding business processes and business process modeling. Information Systems Journal 10: $105-129$

[19] Miers D (1994) Use of tool and technology within a BPR initiative. In: Coulson-Thomas C (ed.): Business Process Re-engineering: Myth and Reality. Elsevier Science, North-Holland, Amsterdam, pp 142-165

[20] Ould MA (1995) Business Processes: Modeling and Analysis for Reengineering \& Improvement. John Wiley, New York

[21] Phalp K, Shepperd M (2000) Quantitative analysis of static models of process. The Journal of Systems and Software 52: 105-112

[22] Phalp K (1998) CAP framework for business process modeling. Information and Software Technology 40: 731-744

[23] Reyneri C (1999) Operational building blocks for business process modeling. Computers in Industry 40: 115-123

[24] Sadiq W, Orlowska EM (2000) Analyzing process models using graph reduction techniques. Information Systems 25: 117-134

[25] Smith H (2003) Business process management - the third wave: business process modeling language $(\mathrm{bpml})$ and its pi-calculus format. Information and Software Technology 45: 1065-1069

[26] Tiwari A (2001) Evolutionary computing techniques for handling variables interaction in engineering design optimization. PhD Thesis, SIMS, Cranfield University, Cranfield, UK

[27] van der Aalst W.M.P, van Hee K.M (1995) Framework for Business Process Redesign. In: JR Callahan (ed.) Proceedings of the Fourth Workshop on Enabling Technologies: Infrastructure for Collaborative Enterprises. IEEE Computer Society Press, Berkeley Springs, pp 36-45

[28] van der Aalst WMP (1996) Petri Net Based Scheduling. OR Spectrum 18: 219-229

[29] van der Aalst WMP (1998) The Application of Petri-Nets to Workflow Management. Journal of Circuits, Systems and Computers 8: 21-66

[30] Zakarian A (2001) Analysis of process models: A fuzzy logic approach. The Int J of Advanced Manuf. Technology 17: 444-452

[31] Zitzler E, Laumanns M, Thiele L (2001) SPEA2: Improving the Strength Pareto Evolutionary Algorithm. TIK Report Nr. 103, Computer Engineer- 
ing and Networks Lab (TIK), Swiss Federal Institute of Technology (ETH) Zurich 